\title{
A tryptophan-based assay method to search regulatory compounds for TCTP
}

Seri Jo ${ }^{\text {a }}$, Eun-Hwa Jang ${ }^{\text {a }}$, Mi-Sun Kim ${ }^{\text {a }}$, Suwon Kim, Kyunglim Lee, and Dong Hae Shin*

College of Pharmacy and Graduates School of Pharmaceutical Sciences, Ewha W. University, Seoul 03760, Korea

${ }^{a}$ These authors contributed equally to this work.

* To whom correspondence should be addressed: Dr. Dong Hae Shin, College of Pharmacy and Graduates School of Pharmaceutical Sciences, Ewha W. University, Seoul 03760, Korea

Tel: 82-2-3277- 4502

Email: dhshin55@ewha.ac.kr

Short title: A tryptophan-based assay for TCTP

The authors have no conflict of interest with any part of this study. 


\begin{abstract}
Transcriptionally controlled tumor protein (TCTP) is a highly conserved protein performing a large number of cellular functions by binding with various partner proteins. The importance of its roles in many diseases requires an assay method to find regulatory compounds. However, the molecular characteristics of TCTP made it difficult to search for chemicals interacting with it. In this study, a tryptophan-based assay method was designed and Y151W mutant TCTP was constructed to search binding chemicals. Since there is no tryptophan in the native sequence of TCTP, the incorporation of tryptophan in the Y151W mutant was very effective to establish the method. A flavonoid library was employed to the assay with the method. With the native and Y151W mutant TCTPs, three flavonoids such as morin, myricetin and isobavachalcone have been found to interact with TCTP. Combined with native gel electrophoresis, the binding region of isobavachalcone was suggested to be the flexible loop of TCTP. This approach can be easily applicable to find binding compounds of proteins with similar molecular characteristics of TCTP.
\end{abstract}

Keywords: Transcriptionally controlled tumor protein (TCTP), Tryptophan, Florescence, Flavonoid, Native gel electrophoresis 


\section{Introduction}

Transcriptionally controlled tumor protein (TCTP) is a highly conserved protein with fundamental cellular functions. It exerts numerous functions by interacting with various partner proteins in many core cell biological processes [1-3]. Especially it demonstrates cytoprotective action in responses to a variety of cellular stresses. In addition, TCTP diversifies its functions depending on oligomeric states that would be triggered by cellular proteolytic digestion or oxidative environment [4]. HRF (Histamine Releasing Factor) is a representative dimeric form activating mast cells and basophils by interacting with a subset of IgE molecules. Paradoxically, the essential cytoprotective function of TCTP also causes it connected to various cancers [5]. Intriguingly, anti-apoptotic activity, promotion of mitosis and anticancer drug resistance of TCTP are all related to the survival of cancer cells. Therefore, if regulatory chemicals of TCTP could be found, various cellular functions mediated by TCTP can be controlled.

To find those compounds, a convenient in-vitro method to assay molecular functions of TCTP should be present. Unfortunately, no direct in-vitro method correlated to molecular functions of TCTP has been reported. One of the reasons is that TCTP is not a catalytic enzyme. Since high-throughput approaches to find out hit chemicals absolutely require easy in-vitro assay methods, TCTP is not applicable. Another reason is the absence of a prominent binding site on the surface of TCTP. Since large cavities on surfaces of proteins increase the binding probability of chemicals and release stabilization energy during interaction with partner molecules, calorimetric techniques can be used to monitor the interaction phenomena. However, there are only two small cavities on the surface of TCTP such as Cavity-1 comprised of $\mathrm{H} 2, \beta 7$ and the loop between $\beta 8$ and $\beta 9$ and Cavity-2 lined up with $\mathrm{H} 3$ and $\beta$-strands $\beta 8, \beta 9, \beta 10$ [6]. Their cavity volumes are around $\sim 360 \mathrm{~A}^{3}$. The small cavity volume implies that common calorimetric methods may be hard to be applied due to the limitation of detecting small binding 
or stabilization energy by instruments. At last, spectroscopic methods are also inapplicable to TCTP due to the absence of tryptophan residues or chromophores.

In order to overcome the above obstruction and to find regulatory chemicals for TCTP, a tryptophan-based assay has been designed. Since there is no tryptophan residue in TCTP, one tryptophan residue was introduced to TCTP by mutation. Subsequently, the binding of chemicals to TCTP could be detected with a general spectrometry method. Since flavonoids have molecular characteristics of both hydrophilicity and hydrophobicity, a flavonoid library was applied to prove the usefulness of the method together with finding binding compounds quickly.

\section{Results and Discussion}

The original hypothesis of general tryptophan-based assay methods is based on the postulation that a change of fluorescence intensity would be detected if chemicals bind to proteins [7]. It is well known that the fluorescence intensity emitted from tryptophan is frequently perturbed by surrounding conditions [8]. In order to test whether the tryptophanbased assay method was feasible to search chemicals interacting with TCTP, one tryptophan residue was incorporated into TCTP. Since there is no tryptophan residue in the original human TCTP sequence, Tyr151 was mutated to Trp151. Tyr 151 was selected because the residue locates where its mutation was expected to influence minimal environmental perturbation. As expected, all the molecular behavior of native f-TCTP and Y151W f-TCTP was almost the same. $\Delta$-TCTP and Y151W $\Delta$-TCTP were also constructed to check if compounds interact with the flexible loop or not. The cell yields harvested for purification of Y151W f-TCTP and Y151W $\Delta$-TCTP were $2.24 \mathrm{~g}$ and $3.7 \mathrm{~g}$ per $1000 \mathrm{ml}$ of $E$. coli culture, respectively. The amount of finally purified proteins were $24.1 \mathrm{mg}$ for the former and $10.8 \mathrm{mg}$ for the latter. For storage 
and assay, both protein solutions were concentrated to $53.1 \mathrm{mg} \mathrm{ml}^{-1}$ and $43.2 \mathrm{mg} \mathrm{ml}^{-1}$, respectively. For the assay, the final concentration was adjusted to $1 \mu \mathrm{M}$ for both cases.

In order to find TCTP regulatory compounds, an in-house flavonoid library was built. Since most flavonoids show a high affinity to proteins due to the presence of hydrophobic aromatic rings and hydrophilic hydroxyl groups, their complementary chemical property endows broad affinities toward proteins. Therefore, a flavonoid library consisting of ten different scaffolds was constructed (Figure 1). It contains three isoflavones, one isoflavane, five flavones, eight flavonols, four flavanols, five flavanones, two flavanonol, one prenylflavonoid, eight chalcones and two unclassified flavonoids (Supplementary Table 1). We applied the library to find flavonoids with affinity to two mutants, Y151W f-TCTP and Y151W $\Delta$-TCTP. Using thirty-nine flavonoids, an inhibitory effect of each compound at $40 \mu \mathrm{M}$ was tested. It has been reported that the flexible loop decreased the inflammatory cytokine activity of full dimeric TCTP (f-dTCTP) [9]. Therefore, using two mutants, we expected to find three different types of chemical compounds according to their binding affinity to; (1) f-TCTP (2) $\Delta$-TCTP (3) fTCTP and $\triangle$-TCTP. If it is possible, this method can be used to find various regulatory compounds controlling TCTP related diseases.

The screening with flavonoids against the in-house flavonoid library was very successful. Among thirty-nine, three flavonoids reduced the flavonoids intensity seriously. Those are morin, myricetin and isobavachalcone (Figure 2). They reduced the fluorescence intensity of both mutants. The three flavonoids were further instigated to check whether they influence the cellular function of TCTP or not. Since TCTP is not a catalytic enzyme, the proof of regulatory functions of flavonoids was performed with TCTP constructs designed to cell experiments. In previous studies, $\mathrm{N}$-terminus truncated TCTP can form dimers $(\triangle \mathrm{N} 11-\mathrm{dTCTP})$ in cells and dimerization of TCTP is necessary for generating its cytokine-like activity [4]. It has also been reported that $\triangle \mathrm{N} 11$-dTCTP stimulates the secretion of proinflammatory cytokines, such as IL- 
8 and GM-CSF in BEAS-2B cells $[4,6]$. Therefore, it is worthwhile to note that the overall architecture of f-dTCTP in-vitro is almost similar to $\triangle \mathrm{N} 11$-dTCTP in-vivo. To verify the cellular function of three flavonoids, each chemical compound was analyzed for their inhibitory effect on $\triangle \mathrm{N} 11$-dTCTP-induced cytokine release. Diosmin, not interacting with two mutant TCTPs was employed as negative control compounds. The secretion of IL-8 was significantly decreased in a dose-dependent manner when cells were treated with various concentrations of three flavonoids (Fig 3. A). Morin, myricetin and isobavachalcone reduced IL-8 secretion. Particularly, myricetin reduced IL- 8 secretion by $26.5 \%, 52.9 \%$ and $77.7 \%$ at $10,20,40 \mu \mathrm{M}$ concentrations, respectively. Isobavachalcone also reduced the IL-8 level to $23.9 \%, 40.9 \%$ and $62.2 \%$, when treated with 1,5 and $10 \mu \mathrm{M}$ of isobavachalcone, respectively. We did not use higher than $10 \mu \mathrm{M}$ of isobavachalcone because of cytotoxicity as shown in Fig 3. B.

In order to independently identify the binding of three flavonoids on TCTP, native gel electrophoresis had been performed. Since TCTP presents as a monomer (f-TCTP) and dimer (f-dTCTP) depending on redox states of Cys172, DTT and tert-Butyl hydroperoxide (tBHP) were pre-treated before electrophoresis, respectively. Under the oxidizing condition, the monomeric and dimeric forms were present (Fig 4. A). Interestingly, three flavonoids, clearly induced the shift of protein bands on the gel implying their interactions with f-dTCTP. Different running patterns of the three flavonoids treated protein bands indicate that the three flavonoids displayed diverse binding modes. Specially, with unknown reasons, the interaction of f-TCTP and morin displayed on the native gel was always smeared and blurred, unlike the interaction with $\Delta$-TCTP. Under the reducing condition, the monomeric form was major on the native gel. The protein bands with all three flavonoids also show irregular patterns in contrast to the monomeric protein band from the control. In both redox states, diosmin not interacting with TCTP did not induce a band shift. The result indicated that three flavonoids seem to 
interact with some parts of the monomeric form of TCTP where are not influenced by dimerization.

In order to check whether the binding regions presumed by the above experiment includes the flexible loop of TCTP or not, native gel electrophoresis had been also performed with $\Delta$ TCTP (Fig 4. B). Since $\Delta$-TCTP also presents as a monomer ( $\Delta$-TCTP) and dimer ( $\Delta$-dTCTP) depending on redox conditions, electrophoresis was carried out under the same redox conditions as above. Under the oxidizing condition, monomer and dimer were present, too. Interestingly, the two flavonoids, morin and myricetin, again clearly induced the shift of protein bands on the gel implying their interactions with $\Delta$-dTCTP. Different running patterns indicate that the binding modes of two flavonoids are diverse. However, isobavachalcone did not interact with $\Delta$-dTCTP. Under the reducing condition, the protein bands of three flavonoids did not introduce any band shift. It clearly indicates that three flavonoids require the flexible loop for their binding with TCTP. In summary, morin and myricetin seemed to interact with some parts of the monomeric form including the flexible loop where are not influenced by dimerization. However, isobavachalcone absolutely required the flexible loop of TCTP for binding. The absence of interaction between isobavachalcone and $\Delta$-dTCTP indicated the strong possibility that isobavachalcone only interacts with the flexible loop of TCTP without overlapping with the main domain of TCTP.

The tryptophan-based assay method employed in this study was very successful. Specially, when combined with the native gel electrophoresis, the accuracy of tryptophan-based method could be confirmed independently. The capability of distinguishing interaction between the flexible loop and chemicals is also the merit of the combined method. Using this approach, a high-throughput method also can be applied to find TCTP regulatory compounds. We hope this method can be used to find binding chemicals against proteins which are none catalytic 
enzymes and have small molecular weight with small narrow cavities and no tryptophan residue.

\section{Materials and Methods}

\subsection{Gene cloning, protein expression and purification for the single mutant $Y 151 W f$ - TCTP}

The pET22b plasmid DNA (pDNA) was used to clone the tctp gene from Homo sapiens (NCBI Ref. seq. NC_000013.11/ GenBank: X16064.1) and its single point mutant. The sequences of the mutagenic primers of Y151W full-TCTP (Y151W f-TCTP) were 5'TGGCATGGTTGCTCTATTGGACTGGCGTGAG GATGGT-3' for the forward strand and 5'-ACCATCCTCACGCCAGTCCAATAGA GCAACCATGCCA-3' for the reverse strand. The polymerase chain reaction (PCR) and transformation were carried out with a QuikChange Lightning Site-Directed Mutagenesis Kit (Agilent Technologies, La Jolla, California, USA) according to the manufacturer's protocol. Transformed single mutant plasmids were purified using a DNA-spin plasmid DNA purification kit (iNtRON Biotechnology, Seoul, Korea).

The cloned plasmid DNA was transformed into E. coli BL21 (DE3) for the mutant expression. E. coli BL21 (DE3) cells were grown on Luria-Bertani (LB) agar plates containing $150 \mu \mathrm{g} \mathrm{ml}^{-1}$ ampicillin. Several colonies were picked and grown in capped test tubes with 10 $\mathrm{ml} \mathrm{LB}$ broth containing $150 \mu \mathrm{g} \mathrm{ml}^{-1}$ ampicillin. A cell stock composed of $0.85 \mathrm{ml}$ culture and $0.15 \mathrm{ml}$ glycerol was prepared and frozen at $193 \mathrm{~K}$ for use in a large culture. The frozen cell stock was grown in $10 \mathrm{ml} \mathrm{LB}$ medium and diluted into $2000 \mathrm{ml}$ fresh LB medium. The culture was incubated at $310 \mathrm{~K}$ with shaking until an $\mathrm{OD}_{600}$ of $0.6-0.8$ was reached. At this point, expression of Y151W f-TCTP was induced using isopropyl- $\beta$-D-1-thiogalactopyranoside (IPTG) at a final concentration of $1 \mathrm{mM}$. The culture was further grown at $310 \mathrm{~K}$ for $3 \mathrm{~h}$ in a shaking 
incubator. Cells were harvested by centrifugation at $7650 \mathrm{~g}\left(6500 \mathrm{rev} \mathrm{min}^{-1}\right)$ for $10 \mathrm{~min}$ in a high-speed refrigerated centrifuge at $277 \mathrm{~K}$. The cultured cell paste $(2.98 \mathrm{~g})$ was resuspended in $25 \mathrm{ml}$ buffer consisting of $50 \mathrm{mM}$ Tris- $\mathrm{HCl} \mathrm{pH} 8.0,100 \mathrm{mM} \mathrm{NaCl}, 10 \mathrm{mM}$ imidazole, 1 mM PMSF and $10 \mathrm{mg} \mathrm{ml}^{-1}$ DNase I. The cell suspension was disrupted using a Digital Sonifier 450 (Branson Ultrasonics Co., Danbury, Connecticut, USA). Cell debris was pelleted by centrifugation at $24900 \mathrm{~g}\left(15000 \mathrm{rev} \mathrm{min}^{-1}\right)$ for $30 \mathrm{~min}$ in a high-speed refrigerated ultracentrifuge at $277 \mathrm{~K}$.

The supernatant was affinity-purified using a $5 \mathrm{ml}$ Hi-Trap His column (GE Healthcare, Piscataway, New Jersey, USA) at room temperature. The column was equilibrated with a buffer consisting of $50 \mathrm{mM}$ Tris- $\mathrm{HCl} \mathrm{pH} 8.0,300 \mathrm{mM} \mathrm{NaCl}, 10 \mathrm{mM}$ imidazole. The target protein was eluted with a buffer consisting of $50 \mathrm{mM}$ Tris- $\mathrm{HCl} \mathrm{pH} 8.0,100 \mathrm{mM} \mathrm{NaCl}$ with a gradient from 10 to $500 \mathrm{mM}$ imidazole. Y151W f-TCTP was further purified by ion-exchange chromatography using a $5 \mathrm{ml} \mathrm{Hi-Trap} \mathrm{Q} \mathrm{column} \mathrm{(GE} \mathrm{Healthcare,} \mathrm{Piscataway,} \mathrm{New} \mathrm{Jersey,}$ USA) equilibrated with a buffer consisting of $20 \mathrm{mM}$ Tris- $\mathrm{HCl} \mathrm{pH} \mathrm{7.0.} \mathrm{The} \mathrm{protein} \mathrm{was} \mathrm{eluted}$ at $0.39 \mathrm{M} \mathrm{NaCl}$ using a linear $\mathrm{NaCl}$ gradient. SDS-PAGE showed one band around $20 \mathrm{kDa}$, corresponding to the molecular weight of full hTCTP_Y151W. The protein was concentrated to $53.1 \mathrm{mg} \mathrm{ml-1}$ for assays in a buffer consisting of $0.39 \mathrm{M} \mathrm{NaCl}$ and $20 \mathrm{mM}$ Tris $\mathrm{pH}$ 7.0.

\subsection{Gene synthesis, protein expression and purification for the single mutant Y151W $\Delta$ - TCTP}

In order to compare the molecular properties of Y151W f-TCTP, the Y151W mutation was also applied to the flexible loop domain (from Arg38 to Val66) deletion mutant, $\Delta$-TCTP. This Y151W $\Delta$-TCTP was synthesized chemically by Bioneer (Daejeon, Korea) and cloned into a bacteriophage T7-based expression vector, $\mathrm{pBT} 7$. The plasmid DNA was transformed into $E$. coli BL21 (DE3) for protein expression. E. coli BL21 (DE3) cells were grown on Luria- 
Bertani (LB) agar plates containing $150 \mu \mathrm{g} \mathrm{ml}^{-1}$ ampicillin. Several colonies were picked and grown in capped test tubes with $10 \mathrm{ml} \mathrm{LB}$ broth containing $150 \mu \mathrm{g} \mathrm{ml}^{-1}$ ampicillin. A cell stock composed of $0.85 \mathrm{ml}$ culture and $0.15 \mathrm{ml}$ glycerol was prepared and frozen at $193 \mathrm{~K}$ for use in a large culture. The frozen cell stock was grown in $5 \mathrm{ml} \mathrm{LB}$ medium and diluted into $1000 \mathrm{ml}$ fresh $\mathrm{LB}$ medium. The culture was incubated at $310 \mathrm{~K}$ with shaking until an $\mathrm{OD}_{600}$ of $0.6-0.8$ was reached. At this point, expression of Y151W $\Delta$-TCTP was induced using isopropyl- $\beta$-D-1-thiogalactopyranoside (IPTG) at a final concentration of $1 \mathrm{mM}$. The culture was further grown at $298 \mathrm{~K}$ for $17 \mathrm{~h}$ in a shaking incubator. Cells were harvested by centrifugation at $7650 \mathrm{~g}\left(6500 \mathrm{rev} \mathrm{min}^{-1}\right)$ for $10 \mathrm{~min}$ in a high-speed refrigerated centrifuge at $277 \mathrm{~K}$. The cultured cell paste $(2.69 \mathrm{~g})$ was resuspended in $25 \mathrm{ml}$ buffer consisting of $50 \mathrm{mM}$ Tris-HCl pH 8.0, 100 mM NaCl, 10 mM imidazole, 1 mM PMSF and 10 mg ml-1 DNase I. The cell suspension was disrupted using a Digital Sonifier 450 (Branson Ultrasonics Co., Danbury, Connecticut, USA). Cell debris was pelleted by centrifugation at $24900 \mathrm{~g}$ (15 000 rev min-1) for $30 \mathrm{~min}$ in a high-speed refrigerated ultra-centrifuge at $277 \mathrm{~K}$.

The supernatant was affinity-purified using a $5 \mathrm{ml} \mathrm{Hi-Trap} \mathrm{His} \mathrm{column} \mathrm{(GE} \mathrm{Healthcare,}$ Piscataway, New Jersey, USA) at room temperature. The column was equilibrated with a buffer consisting of $50 \mathrm{mM}$ Tris $-\mathrm{HCl} \mathrm{pH} 8.0,300 \mathrm{mM} \mathrm{NaCl}, 10 \mathrm{mM}$ imidazole. The target protein was eluted with a buffer consisting of $50 \mathrm{mM}$ Tris- $\mathrm{HCl} \mathrm{pH} 8.0,100 \mathrm{mM} \mathrm{NaCl}$ with a gradient from 10 to $500 \mathrm{mM}$ imidazole. Y151W $\Delta$-TCTP was further purified by ion-exchange chromatography using a $5 \mathrm{ml}$ Hi-Trap Q column (GE Healthcare, Piscataway, New Jersey, USA) equilibrated with a buffer consisting of $20 \mathrm{mM}$ Tris- $\mathrm{HCl} \mathrm{pH} \mathrm{7.5.} \mathrm{The} \mathrm{protein} \mathrm{was} \mathrm{eluted}$ at $0.3 \mathrm{M} \mathrm{NaCl}$ using a linear $\mathrm{NaCl}$ gradient. SDS-PAGE showed one band around $18 \mathrm{kDa}$, corresponding to the molecular weight of Y151W $\Delta$-TCTP. The protein was concentrated to 4 $3.2 \mathrm{mg} \mathrm{ml}-1$ for assays in a buffer consisting of $0.3 \mathrm{M} \mathrm{NaCl}$ and $20 \mathrm{mM}$ Tris $\mathrm{pH}$ 7.5. 


\subsection{Absorption spectroscopic studies based on the incorporated tryptophan}

The fluorescence spectra of Y151W f-TCTP and Y151W $\Delta$-TCTP with chemical compounds were investigated. The fluorescence measurements were recorded with a SpectraMax i3x Multi-mode microplate reader (Molecular Devices) at excitation and emission wavelengths of $280 \mathrm{~nm}$ and $310-500 \mathrm{~nm}$, respectively. Both of them showed a strong fluorescence emission with a peak at $320 \mathrm{~nm}$ at the excitation wavelength of $280 \mathrm{~nm}$, unlike native TCTP. In contrast, the chemical compounds were almost non-fluorescent under the same experiment condition. Each $40 \mu \mathrm{M}$ chemical compound was incubated with each mutant for 1 hour and the fluorescence intensity of each solution was measured.

\subsection{Measurement of IL-8 secretion}

The human bronchial epithelial BEAS-2B cells $\left(2 \times 10^{4}\right.$ cells/well $)$ were cultured in 48 -well plates. Chemical compounds $(1,5,10 \mu \mathrm{M})$ and 1-10 residues deleted rat recombinant dimeric TCTP (Del-N11dTCTP) $(8 \mu \mathrm{g} / \mathrm{ml})$ were pre-incubated for $15 \mathrm{~min}$ and treated to each well as previously described [4]. After 16-18 h, the supernatant was collected and IL-8 concentration was measured by enzyme-linked immunosorbent assay (ELISA) according to the manufacturer's instructions (Biolegend, San Diego, CA, USA).

\subsection{Cell viability assay}

BEAS-2B cells $\left(5 \times 10^{3}\right.$ cells/well $)$ were seeded in 96 -well plates. The cultured cells were treated with various concentrations of indicated chemical compounds for $24 \mathrm{~h}$. Subsequently, the 3-(4,5-dimethylthiazol-2-yl)-2,5 diphenyl tetrazolium bromide (MTT) solution (Sigma) was added and incubated for $3-4 \mathrm{~h}$ at $37^{\circ} \mathrm{C}$. After incubation, the supernatant was removed, and formazan crystals were dissolved in DMSO (100 $\mu \mathrm{l} /$ well). Absorbance was read at $570 \mathrm{~nm}$ 
using a microplate reader and the viability of cells was expressed as a percentage relative to the control cells.

\section{Disclosure statement}

The authors declare no conflict of interest.

\section{Funding}

This work was supported by the Basic Science Research Programs, 2018R1D1A1B07050781 to DHS and 2018R1D1A1B07050942 to MK, funded by the National Research Foundation of Korea grant granted by the Ministry of Education, Science and Technology, Republic of Korea (MEST). S. Jo was supported by Brain Korea 21 (BK21) Project.

\section{Reference}

[1] Tueson, D. O., Speck, L. S., Lett-Brown, M. A. \& Grant, J. A. Histamine-releasing activity (HRA). II. Interaction with basophils and physicochemical characterization. J. Immunol. 1979, $123,633-639$.

[2] Tueson, D. O., Speck, L. S., Lett-Brown, M. A. \& Grant, J. A. Histamine-releasing activity (HRA). I. Production by mitogen- or antigen-stimulated human mononuclear cells. J. Immunol. 1979, 123, 626-632.

[3] MacDonald, S. M., Rafnar, T., Langdon, J. \& Lichtenstein, L. M. Molecular identifcation of an IgE-dependent histamine-releasing factor. Science. 1995, 269, 688-690.

[4] Kim, M. et al. Dimerization of translationally controlled tumor protein is essential for its $\begin{array}{llll}\text { cytokine-like } \quad \text { activity. } & \text { PLoS 2009, } & \text { e6464, }\end{array}$ https://doi.org/10.1371/journal.pone.0006464 
[5] Bhisutthibhan, J. et al. Te Plasmodium falciparum translationally controlled tumor protein homolog and its reaction with the antimalarial drug artemisinin. J. Biol. Chem. 1998, 273, 16192-16198.

[6] Lee, H., Kim, M., Lee, J. et al. Flexible loop and helix 2 domains of TCTP are the functional domains of dimerized TCTP. Sci Rep. 2020, 10, 197. https://doi.org/10.1038/s41598-01957064-9

[7] Ghisaidoobe ABT, Chung SJ. Intrinsic Tryptophan Fluorescence in the Detection and Analysis of Proteins: A Focus on Förster Resonance Energy Transfer Techniques. International Journal of Molecular Sciences. 2014, 15, 12, 22518-22538.

[8] Adams, Paul D et al. "Intramolecular quenching of tryptophan fluorescence by the peptide bond in cyclic hexapeptides." Journal of the American Chemical Society vol. 2002, 124, 31 : 9278-86. doi:10.1021/ja0167710

[9] Kim M, Min HJ, Won HY, Park H, Lee JC, Park HW, Chung J, Hwang ES, Lee K. Dimerization of translationally controlled tumor protein is essential for its cytokine-like activity. PLoS One. 2009, 31;4, 7 :e6464. doi: 10.1371/journal.pone.0006464. PMID: 19649253; PMCID: PMC2715101.

\section{Figure Legends}




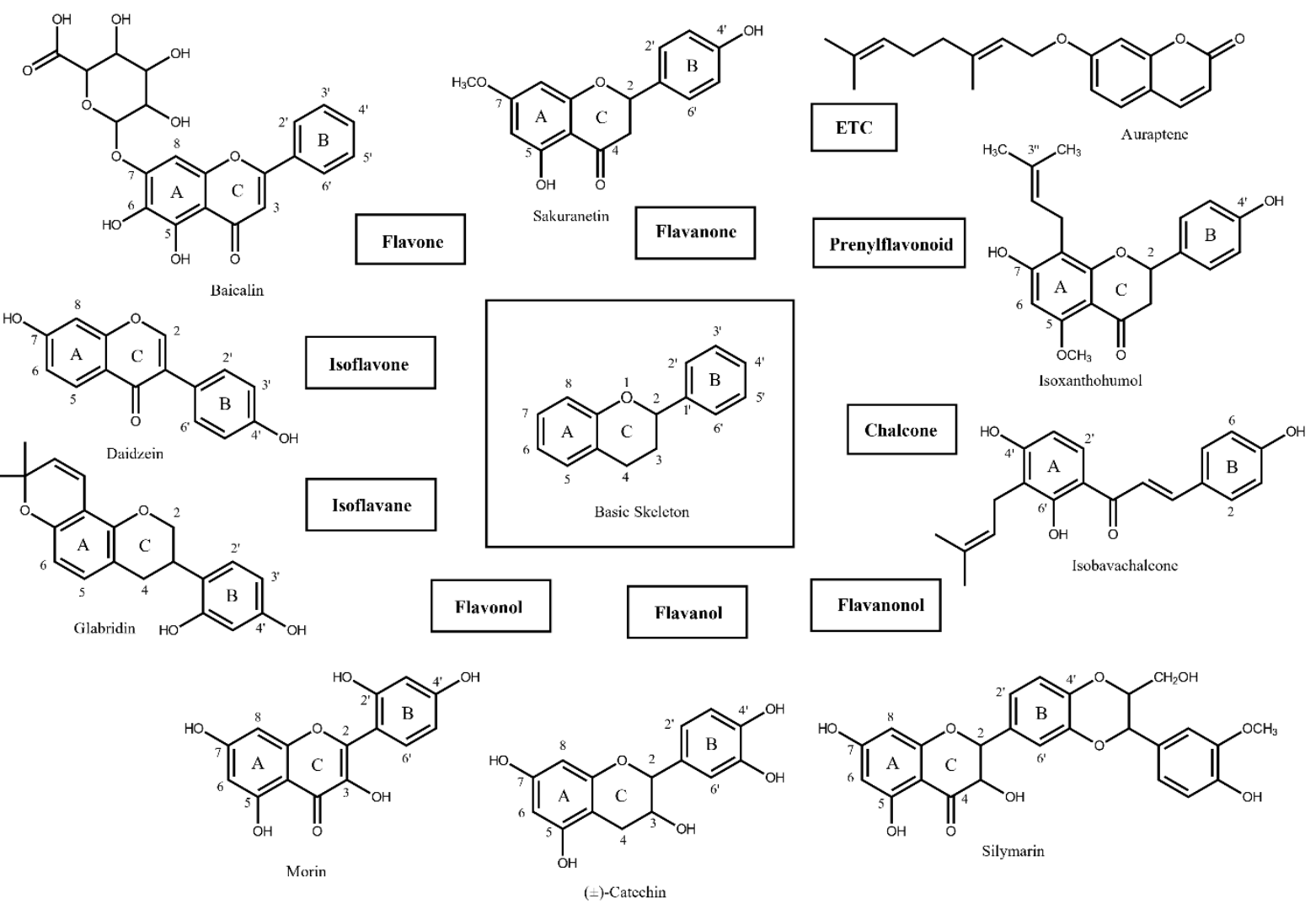

Figure 1. The basic skeleton structures of flavonoids and their scaffolds. Basic representative structures of the most common flavonoids classified in this study were drawn with rings and numbered positions. 
Y151W f-TCTP

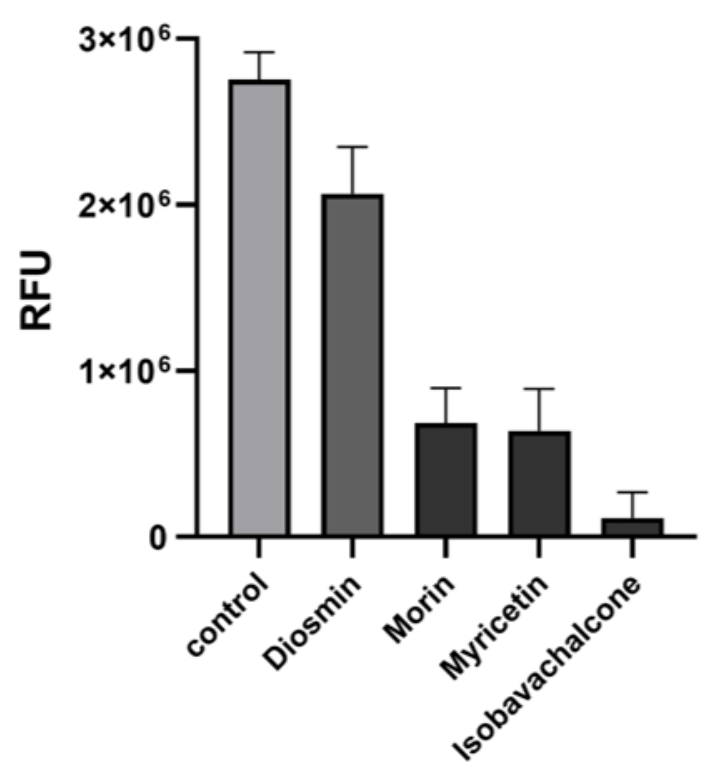

Y151W $\Delta$-TCTP

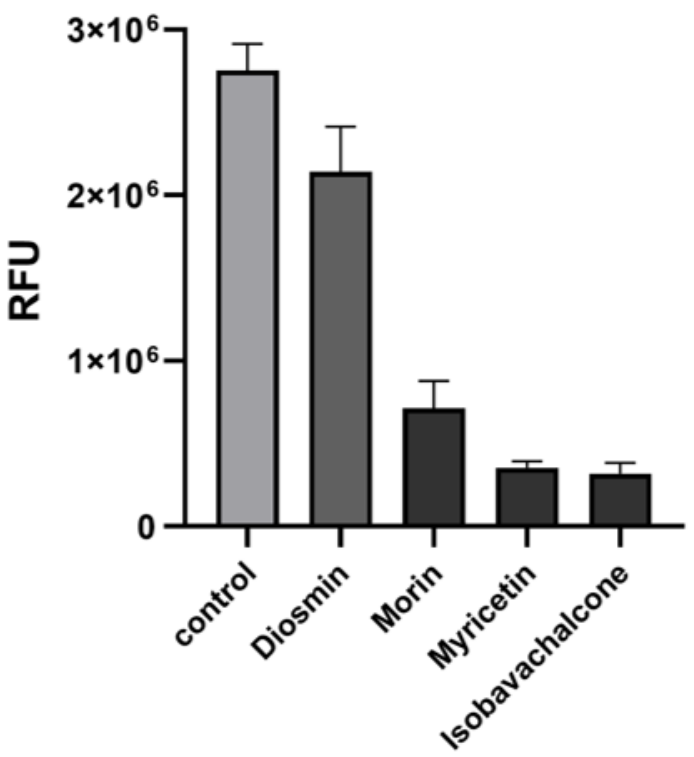

Figure 2. Fluorescence quenching of tryptophan incorporated TCTP mutants. Each bar represents the fluorescence emission at $320 \mathrm{~nm}$. The first gray bar represents the control. Each bar is expressed as the mean \pm standard error of the mean $(n=3)$. RFU $=$ Relative Fluorescence Units

(A)

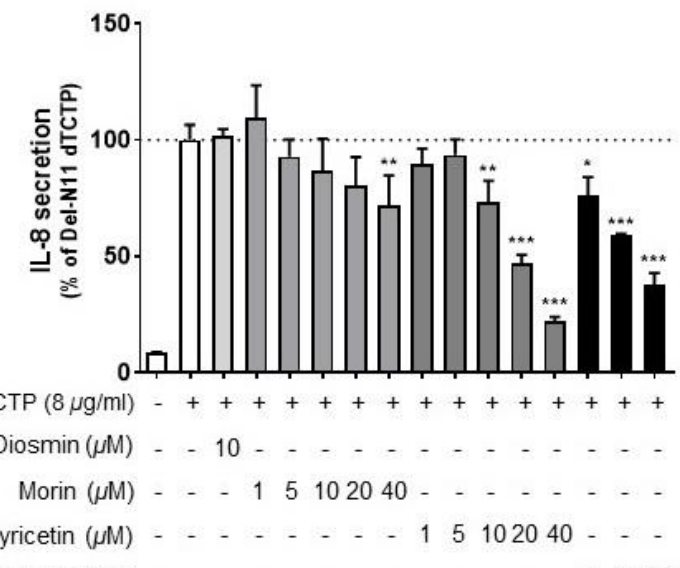

Isobavachalcone $(\mu \mathrm{M})$
(B)

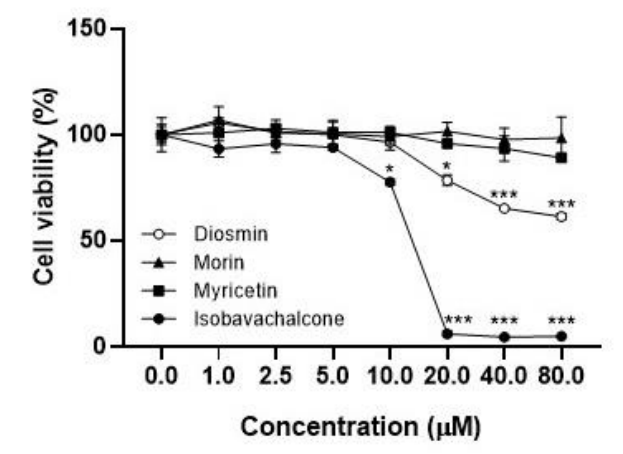

Figure 3. The effect of three flavonoids on BEAS-2B cells activity and viability. (A) Cells 
were treated with various concentrations of chemical compounds and $8 \mu \mathrm{g} / \mathrm{ml}$ of $\Delta \mathrm{N} 11$-dTCTP for 16-18 h. The IL-8 in the supernatant was quantified by ELISA. Data are presented as mean $\pm \mathrm{SD}, \mathrm{n}=3 \cdot \mathrm{p}^{*}<0.05, \mathrm{p}^{* *}<0.01, \mathrm{p}^{* * *}<0.001 ;$ compared to $\Delta \mathrm{N} 11-\mathrm{dTCTP}$ treated group. (B) Cells were incubated with each compound $(0-80 \mu \mathrm{M})$ for $24 \mathrm{~h}$ and cell viability was determined by MTT assay. Data are presented as mean $\pm \mathrm{SD}, \mathrm{n}=3 . \mathrm{p}^{*}<0.05, \mathrm{p}^{* * *}<0.001$; compared to control.

(A) f-TCTP
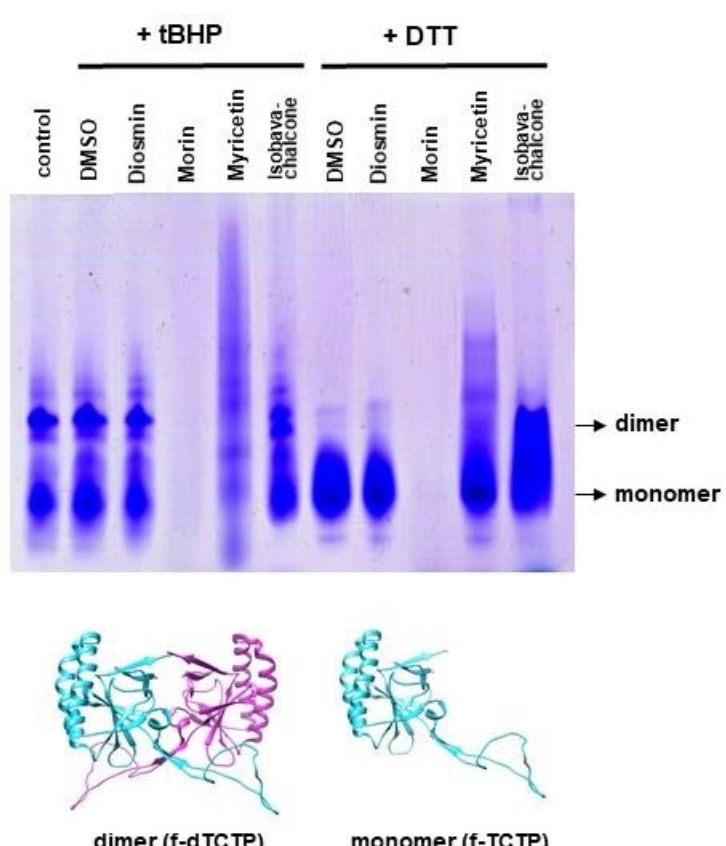

dimer (f-dTCTP)

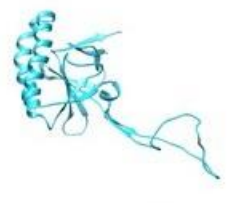

monomer (f-TCTP)
(B) $\triangle$-TCTP
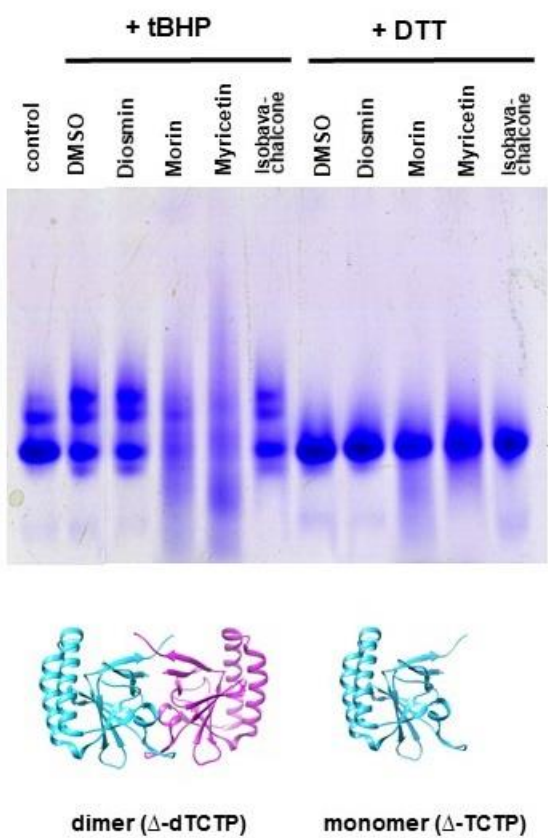

monomer $(\Delta-\mathrm{TCTP})$

Figure 4. Native gel runs after treating redox agents against f-TCTP and $\triangle$-TCTP. (A)

Dimer of f-TCTP is formed with tBHP and monomer with DTT. The effect of DMSO on the native gel runs was also compared. Morin treated f-TCTP always did not display any protein band but showed an evenly smeared and blurred pattern. The crystal structures of f-TCTP according to redox states are depicted on the bottom. (B) Dimer of $\Delta$-TCTP is formed with 
tBHP and monomer with DTT. The crystal structures of $\Delta$-TCTP according to redox states are depicted on the bottom.

\section{Supplementary Table 1. A Flavonoid Library}

*Isoflavone,1-3; Isoflavane,4; Flavone,5-9; Flavonol,10-17; Flavanol,18-21; Flavanone,22-26; Flavanonl,27-28; Prenylflavonoid,29; Chalcone,30-37; unclassified,38-39

\begin{tabular}{|c|c|c|c|}
\hline No & Name of compound & No & Name of compound \\
\hline 1 & Daidzein & 21 & $( \pm)$-Catechin \\
\hline 2 & Genistein & 22 & Hesperidin \\
\hline 3 & Genistin & 23 & Naringenin \\
\hline 4 & Glabridin & 24 & Sakuranetin \\
\hline 5 & Baicalein & 25 & Naringin \\
\hline 6 & Diosmin & 26 & Poncirin \\
\hline 7 & Diosmetin & 27 & $( \pm)$-Taxifolin hydrate \\
\hline 8 & Skullcapflavone II & 28 & Silibinin \\
\hline 9 & beta-Naphthoflavone & 29 & Isoxanthohumol \\
\hline 10 & Herbacetin & 30 & Isobavachalcone \\
\hline 11 & Kaempferol & 31 & $2,2^{\prime}, 4^{\prime}$-Trihydroxychalcone \\
\hline 12 & Morin & 32 & Dienestrol \\
\hline 13 & Myricetin & 33 & Sofalcone \\
\hline 14 & Fisetin & 34 & Rhodamine $6 \mathrm{G}$ \\
\hline 15 & Quercitrin & 35 & FCLA Free Acid \\
\hline 16 & Quercetin & 36 & Helichrysetin \\
\hline 17 & Quercetin 3- $\beta$-D-glucoside & 37 & Cardamonin \\
\hline 18 & (-)-Gallocatechin & 38 & Mangiferin \\
\hline 19 & (-)-Epigallocatechin gallate & 39 & Auraptene \\
\hline 20 & $(-)$-Epicatechin & & \\
\hline
\end{tabular}

